\title{
DESENVOLVIMENTO DA MENTALIDADE BIM: ESTUDO DE CASO DE APLICAÇÃO PEDAGÓGICA DE ESCRITÓRIO DE PROJETOS
}

\author{
Lissa Araújo $^{(1)}$, Roberta Oliveira ${ }^{(1)}$, Michele Carvalho ${ }^{(1)}$ \\ (1) Universidade de Brasília, Brasília - Distrito Federal - Brasil
}

\begin{abstract}
Resumo
A apresentação do processo BIM (Building Information Modelling) para a indústria da construção civil primordialmente foca na utilização de novos softwares. Entretanto, o BIM sugere uma mudança mais profunda de comunicação e integração de informações, visando habilitar o trabalho conjunto de um número de disciplinas, assim como níveis crescentes de coordenação e colaboração. Essas mudanças, porém, necessitam aceitação e adaptação das partes interessadas. O objetivo deste trabalho é colaborar com a análise dos desafios comportamentais a serem superados pelas equipes que aplicam o BIM no desenvolvimento de projetos. Um estudo de caso foi realizado na disciplina de graduação do curso de Engenharia Civil "Planejamento e Controle de Construções", em que a turma, dividida em grupos, aplicou o processo BIM para a modelagem de um projeto. Os resultados foram insatisfatórios em termos de produto, por conta de errônea divisão de tarefas, falta de compartilhamento de informações entre as partes e compatibilização das etapas, mas foram satisfatórios em termos de aprendizado, por conclusão dos mesmos ao fim da atividade e considerando que este foi o primeiro contato da maioria dos alunos com o BIM. Este trabalho contribui para a discussão do verdadeiro foco do processo BIM, que vai além dos softwares e processamento das máquinas: a implementação efetiva de uma rede de informações e contribuições técnicas entre as equipes de um projeto.
\end{abstract}

\section{Introdução}

A indústria da construção civil está sempre buscando novas formas de se tornar mais eficiente. $\mathrm{O}$ BIM (Building Information Modelling) aparece na vanguarda do desenvolvimento tecnológico e metodológico, e promete revolucionar o mercado [1]. Ele apresenta-se como um processo virtual que possibilita a reunião de todas as disciplinas, sistemas e aspectos de uma obra em um único modelo, permitindo uma colaboração mais estreita dos agentes de projeto e incentivando a integração das funções de todas as partes interessadas. É uma tecnologia que auxilia a visualização do que deve ser construído em um ambiente simulado para identificar 
possíveis problemas [2], focando na redução das ineficiências e aumentando a qualidade e rentabilidade dos processos [3].

A apresentação do processo BIM e de suas possibilidades para a indústria da construção civil, apesar de primordialmente focar na utilização de softwares que dão suporte a esse modelo de trabalho, implica uma mudança mais profunda: as partes interessadas são chamadas a manter um fluxo de informações integrado e uma visão sistêmica e crítica de todo o processo, desde a concepção até a entrega do produto final.

Porém, alguns obstáculos já foram registrados antes da plena aceitação dessa nova forma de trabalho, especialmente junto a colaboradores com habilidades tradicionais [4]. Surge a necessidade de se descodificar um novo vocabulário, de aprender novas ferramentas e de implementar novos procedimentos [5]. Além disso, empresas apontam a formação de profissionais como uma etapa imprescindível, porém muitas vezes onerosa e trabalhosa [2;58]. A contratação de profissionais na área de construção que já possuam conhecimentos e competências para colaborar e comunicar através das ferramentas BIM são mais atrativas. $\mathrm{O}$ BIM, portanto, deve ser introduzido no currículo da graduação como uma evolução natural das ferramentas de visualização e comunicação, seja explicitamente, através de unidades curriculares de modelagem ou desenho, seja implicitamente, através do apoio continuado a todas as unidades curriculares ao longo do curso [9].

O objetivo deste trabalho é aprofundar as percepções acerca da mentalidade BIM em desenvolvimento no mercado e da necessidade de treinamento dos engenheiros civis a nível de graduação por meio de um estudo de caso. O ensino da disciplina "Planejamento e Controle de Construções" do curso de graduação em Engenharia Civil da Universidade de Brasília (UnB) por meio de uma aplicação pedagógica de um escritório de projetos foi analisado, em que a turma, dividida em grupos de trabalho, operou quatro fases essenciais da modelagem de um projeto, aplicando o processo BIM.

\section{O ensino do BIM nos cursos de Engenharia Civil e Arquitetura}

Muitas grades curriculares de graduações em Engenharia Civil no Brasil ainda mantém o ensino dos métodos tradicionais de desenho em CAD, ou associam o ensino do BIM à utilização de algum software exclusivamente. Outras possuem uma disciplina específica sobre BIM, porém optativa. Existem ainda as que associam essa temática a cursos de pós-graduação, dando ao BIM uma imagem de complexidade ou sofisticação quando comparado à habilidade em CAD [10]. A Universidade de Brasília, por exemplo, oferece uma disciplina optativa no curso de Engenharia Civil voltada para o ensino do processo BIM, incentivando o desenvolvimento da mentalidade e maturidade associadas à aplicação de ferramentas. No entanto, por não ser obrigatória, a aderência pelos alunos é pequena e por vezes se restringe aos que estão no último período do curso, critério adotado para desempate na disputa por vagas.

Em relação às tendências no cenário internacional quanto ao ensino de BIM, duas formas de ensino predominam: uma ou duas disciplinas no início ou final do curso, ou várias matérias no currículo como recurso didático. Pondera-se ainda que as estratégias de ensino e aprendizagem de BIM podem variar de acordo com o nível de competência que se deseja alcançar [11]. As realidades didáticas nacionais mostram uma implantação gradual e pouco efetiva nos cursos de Arquitetura e Engenharia Civil quando comparado ao cenário internacional, que apresenta maior amadurecimento e envolvimento de mais de uma disciplina em vários momentos da 
formação [12]. Além da alteração de currículo nos cursos, também deve ser exigida a capacitação e aceitação dos professores quanto à utilidade dessa tecnologia, e investimento em infraestrutura [13-14].

O ensino de BIM no âmbito brasileiro também vem sendo estimulado pela realização de eventos como o Encontro Nacional sobre o ensino de BIM (ENEBIM), em sua segunda edição em 2019, permitindo a divulgação e compartilhamento de experiências didáticas e conteúdos desenvolvidos. Os registros mostram a implementação dos conceitos BIM em disciplina experimental na pós-graduação [15], pós-graduação Lato Sensu aplicado em projetos de infraestrutura [16], metodologia ativa interdisciplinar na graduação que promove a integração de todas as disciplinas aplicadas no semestre vigente [17], inserção de conteúdos BIM dentro de um curso técnico de Edificações [18], e inclusive parcerias entre empresas e universidade para fornecimento de softwares e treinamentos [19].

Apesar das preocupações em vários âmbitos da educação a nível acadêmico, a abordagem de BIM no ensino deve ir além da capacitação instrumental oferecida pelas disciplinas de informática aplicada [12]. A inserção do BIM nos processos da construção civil é reconhecidamente gradativa e depende de alguns fatores já mapeados [20], detalhados a seguir.

\section{Mentalidade e maturidade BIM}

A despeito da sugestão de modernidade do BIM, investigadores já discutem os impactos de modelos de construção desde antes da criação do termo Building Information Modelling. As promessas de mudanças sugeridas pelo processo BIM incluem várias teorias organizacionais, informacionais e regulatórias, portanto, a necessidade de ordenar tal conhecimento em domínios já se faz evidente a algum tempo [20,21].

Sendo essa uma necessidade a muito mapeada, vários modelos de framework já existem em constante melhoria. Em termos de implantação, especificamente, foi cunhado o termo "Estágios BIM", nos quais são delineados os níveis de maturidade de implementação, iniciando com o nível zero, ou seja, anterior a uma efetiva prática do modelo, passando por três estágios fixos, em que a estrutura organizacional da empresa está perfeitamente delimitada com responsabilidades e atividades BIM, e na qual as lições aprendidas são documentadas e melhoradas a cada novo projeto [22]. O estágio final é variável a fim de absorver futuros e ainda desconhecidos avanços nesse campo, já que o BIM tem um aspecto inovador e precisa acolher adaptabilidade em seus conceitos [23].

O nível de maturidade BIM depende, entre outros aspectos, das pessoas e da cultura envolvida. A motivação a nível empresarial, de gerar economia e competitividade, deve estar em sintonia com a motivação a nível pessoal, ou seja, a vontade própria dos mesmos de trabalhar com a ferramenta e expandir seus conhecimentos, levando a uma atitude colaborativa entre os integrantes da cadeia produtiva. Alcançar uma abordagem integrada e multidisciplinar BIM requer uma atitude voltada à cooperação, e uma vontade de mudar inerente à cultura da empresa, suas estruturas e processos [22], seguindo então a premissa do trabalho colaborativo, no qual o esforço de um pode ser aproveitado por outro [24].

Portanto, a implementação não é apenas física. É crucial perceber que o BIM implica mais do que mudar softwares em escritórios. O surgimento de uma nova tecnologia implica uma nova forma de pensar e projetar. Então, o BIM requer uma nova mentalidade, o que leva a novas 
responsabilidades e novos cargos. O impacto causado pelo BIM precisa refletir o impulso mental causado pela mudança de paradigmas computacionais na era moderna [21].

Por isso, torna-se necessário superar o receio dos profissionais que não compartilham dessa concepção de trabalho, e torna-los não apenas aptos, mas confiantes no processo BIM e nos seus benefícios.

\section{Estudo de Caso: Escritório de Projetos em BIM}

Após explanação da importância do processo BIM para o mercado e a necessidade de fomentar sua mentalidade nos profissionais da construção civil em desenvolvimento, foi proposta uma atividade pedagógica de simulação de um escritório de projetos na disciplina de "Planejamento e Controle de Construções" pertencente à grade curricular do oitavo período do curso de graduação de Engenharia Civil da Universidade de Brasília (UnB), com 60 horas. A atividade buscou desenvolver habilidades e competências em softwares, e também a necessidade de retroalimentação e revisão constante das etapas em um trabalho colaborativo.

A matéria contou com 44 alunos matriculados, divididos em grupos fixos, com 4 membros, durante toda a disciplina. Inicialmente, os alunos executaram uma pesquisa bibliográfica sobre os assuntos pertinentes à aplicação do processo BIM, assim como analisaram material didático sugerido. Posteriormente, foram aplicados os conceitos discutidos em um projeto real com as fases de modelagem arquitetônica, orçamentação, projeto de canteiro e planejamento da obra. Todas as fases do projeto foram executadas por todos os grupos, e eles utilizaram seu próprio resultado da fase anterior como informação precedente para a fase seguinte. Os intervalos de fase também foram marcados por avaliações de desempenho. Em cada uma das etapas, buscouse a utilização de softwares com interface BIM que possuem versão teste ou gratuita para estudantes, e que fornecem relatórios compatíveis com o padrão brasileiro. A Figura 1 mostra a sequência de atividades desenvolvidas, e os softwares utilizados.

Os alunos foram supervisionados pela professora ministrante da disciplina, doutora em estruturas e construção civil com ênfase em gestão e sustentabilidade de obras, e por dois orientandos a nível de mestrado da mesma. Os professores buscaram orientar os alunos de forma a ajudá-los a superar a barreira inicial da falta de familiaridade com o modelo de trabalho e as ferramentas, mas respeitando a autonomia de aprendizado dos estudantes e as sugestões apresentadas.

$\mathrm{Na}$ primeira fase, os alunos tiveram acesso a um projeto arquitetônico em formato $d w g$ e precisaram modelá-lo na plataforma Revit, se preocupando com a compatibilidade com o Memorial Descritivo (MD) e também com a Estrutura Analítica de Projeto (EAP), além de inserção dos parâmetros corretos de cada elemento no Revit para possibilitar a extração de quantitativos para a orçamentação.

O orçamento foi realizado com o auxílio do software brasileiro OrçaFascio, atribuindo composições unitárias a cada serviço previsto na EAP, com a extração de quantitativos pelo plug-in OrçaBIM, que permite a quantificação a partir do modelo desenvolvido no Revit. Foram gerados então os orçamentos sintético e analítico, além das curvas $\mathrm{ABC}$ de insumo e de serviços. 


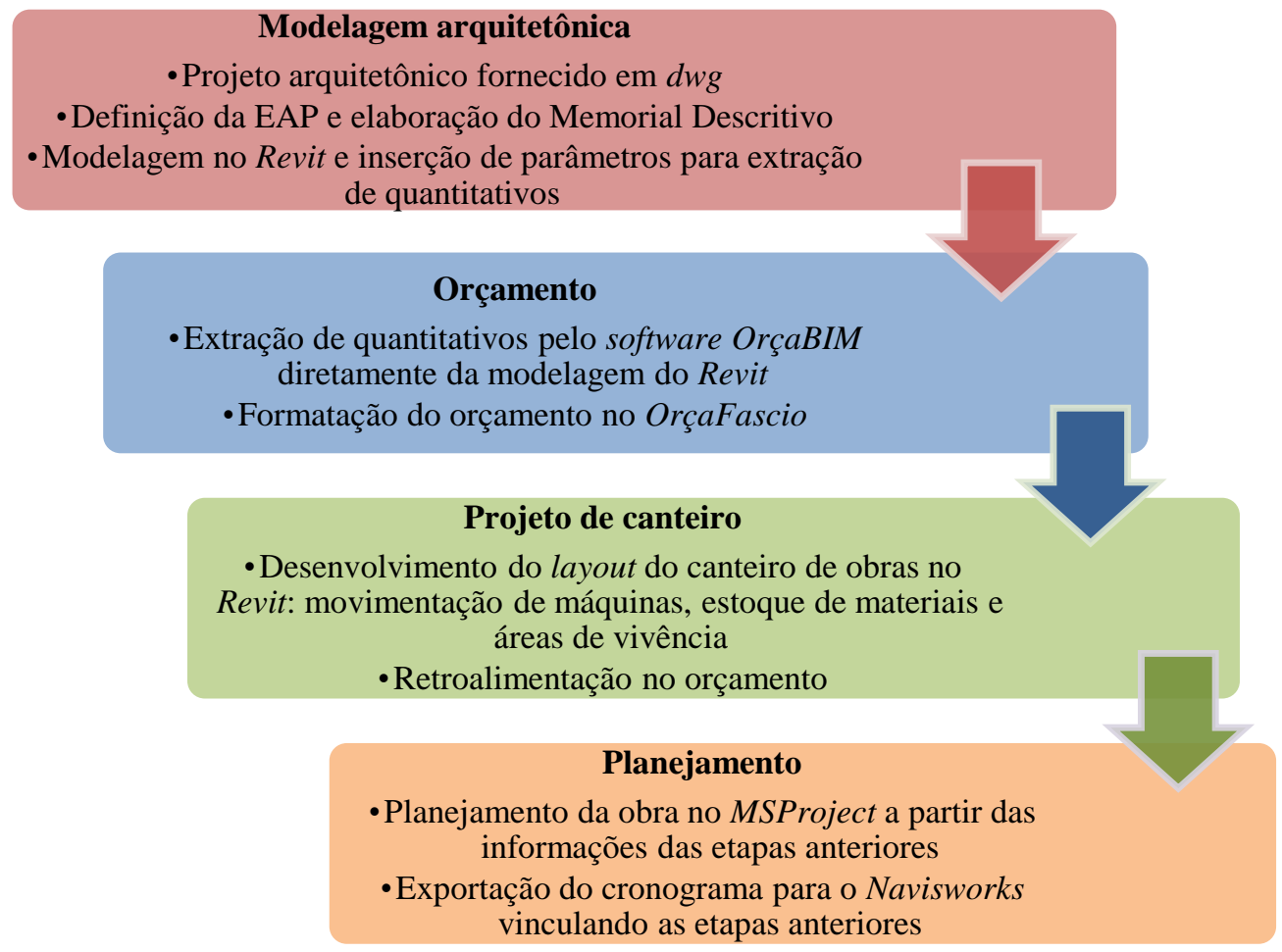

Figura 1: Fases de projeto operadas na simulação.

Para a fase de projeto de canteiro, solicitou-se o desenvolvimento do layout do canteiro de obras no Revit para uma etapa simplificada da obra, entre as opções: serviços preliminares, estrutura, acabamento, etc, com a previsão do fluxo de movimentação dos equipamentos e das áreas de estoque de materiais e de vivência (refeitório, banheiro, escritório, entre outros). Tais itens puderam ser previstos e dimensionados a partir dos produtos da entrega anterior do próprio grupo, que já prognosticavam a quantidade de material, mão-de-obra e equipamento.

Por fim, os alunos montaram o cronograma da obra no programa MSProject com a aplicação das técnicas de planejamento e controle discutidos em sala. A cada atividade concluída, os grupos apresentaram os resultados obtidos aos demais, professores e colegas. Cada apresentação foi seguida de autoavaliações orais do processo de aprendizagem, além do feedback quanto aos pontos de melhoria e à necessidade de retroalimentação das etapas anteriores, inerente ao processo BIM.

\section{Resultados e discussões}

Anteriormente ao desenvolvimento dos produtos, não houve análise formal do conhecimento em BIM dos alunos, visto que este conhecimento não constitui exigência para cursar a disciplina. Durante o desenvolvimento dos produtos, porém, notou-se pouca familiaridade dos mesmos quanto à utilização dos softwares, por observação dos professores e feedback dos alunos quanto tal conteúdo foi exposto. Foi detectado um conhecimento mediano quanto à utilização do Revit e MSProject, e baixo conhecimento quanto ao processo de orçamentação em BIM, existência do Navisworks ou das suas ferramentas para acompanhamento. 
Especificamente quanto ao uso do Revit, poucos alunos tinham conhecimento da modelagem parametrizada (por exemplo, a atribuição de diferentes camadas e espessuras para o componente "parede" do modelo). Devido ao curto tempo, os alunos não conseguiram realizar a exportação do cronograma para o programa Navisworks, entretanto, foi feita uma demonstração na plataforma, com a vinculação da modelagem no Revit, do cronograma desenvolvido e do lançamento do custo de cada etapa construtiva.

Os grupos optaram pela divisão de tarefas entre os componentes, o que provocou falhas de comunicação interna pela ausência de compatibilização das informações antes da entrega dos produtos. Muitos definiram corretamente a EAP para o orçamento, mas não mantiveram a mesma divisão dentro do MD, dificultando o entendimento do documento. Outros previram materiais no orçamento que estavam com outra especificação no MD, que por sua vez estavam diferentes na modelagem no Revit. Porém, o levantamento de quantitativos pelo OrçaBIM proporcionou um entendimento melhor quanto ao nível de detalhamento necessário na modelagem para a quantificação correta. Durante a fase de definição e dimensionamento do canteiro, e também do planejamento da obra, muitos tiveram dificuldade de entender que o orçamento já previa a mão-de-obra e equipamentos dentro das composições unitárias e que o correto seria utilizá-lo para a definição das equipes, sendo necessários apenas ajustes para a compatibilização entre as etapas.

Já nas apresentações, tomados como pontos de checagem, enfatizou-se a necessidade de retroalimentação constante do orçamento e atualização do planejamento, e se necessário, modificação da modelagem em concordância. Os alunos perceberam a real utilidade do processo BIM: permitir a atualização mais ágil de todos os produtos de um projeto quando detectadas modificações necessárias. Uma modificação em uma área, ou mesmo um erro detectado, impacta todas as equipes e mostra a utilidade de um fluxo de informações eficiente, não só dentro de uma mesma equipe, mas com todas as áreas envolvidas no processo, evitando assim retrabalhos.

Por fim, notou-se uma evolução da maioria dos alunos ao longo do semestre, pois foram percebidos diminuição de erros, amadurecimento dos conceitos e entendimento do processo BIM. O feedback foi positivo, de todos os envolvidos na disciplina, com ressalvas quanto à necessidade de aumento da carga horária para um melhor aproveitamento.

As dificuldades encontradas na implementação BIM durante a simulação do escritório de projetos variaram desde, inicialmente, (i) falta de familiaridade com os softwares disponíveis e suas ferramentas, passando pela (ii) correta e útil estruturação dos processos e documentos gerados ao longo das etapas, até (iii) a percepção da necessidade de retroalimentação dos produtos parciais e mudanças interdependentes. Os alunos trouxeram, para a atividade, concepções tradicionais do processo de projeto quando não previram a necessidade de retroalimentação e comunicação efetiva entre as partes, mesmo depois de conhecerem os conceitos e softwares, mostrando que a mudança de mentalidade deve vir antes da prática de modelagem integrada. Percebe-se então a abrangência da implementação BIM, indo além do uso isolado dos softwares, ou o "little BIM", mas efetivamente dando suporte ao método de trabalho e à mentalidade, chamado "BIG BIM" [21].

Os alunos puderam ver na prática todos os impactos que a iniciativa provoca ao longo de toda a cadeia de projeto, constatando que a adoção do processo BIM implica um aumento do escopo e das responsabilidades dos projetistas e engenheiros a nível de concepção de projeto, e da real necessidade de se aumentar o esforço, aprendizado e investimento [25]. 


\section{Conclusões}

O objetivo deste trabalho foi contribuir com a discussão sobre a aceitação do processo BIM pelas partes de um projeto, e de como o conhecimento dessa ferramenta deve ir além dos softwares. É preciso embutir nos profissionais a mentalidade BIM: a necessidade de cooperação e comunicação, para que o fluxo de informações se torne eficiente e o processo seja realmente unificado.

A aplicação pedagógica de um escritório de projetos em uma disciplina da graduação se provou uma boa ferramenta para demonstrar a necessidade de cooperação e comunicação em um caso prático. Foi possível demonstrar a importância da compatibilização de informações e produtos, às custas da qualidade de projeto e da necessidade de retrabalhos. Além disso, o processo BIM promoveu a interdisciplinaridade, essencial em um curso como a Engenharia Civil.

Contudo, a simulação não teve sucesso completo. Não foi possível trabalhar o software Navisworks devido ao tempo reduzido. Os resultados foram insatisfatórios também em termos de produto, porque estes exibiram excessiva falta de compatibilização durante as entregas parciais, e as causas variam de uma errônea divisão de tarefas à falta de alinhamento de informações entre as partes. No entanto, é preciso considerar que a carga horária da disciplina limitou a exposição prolongada a um conhecimento tão complexo, e a maioria dos alunos estava tendo o primeiro contato com o BIM. Portanto, avalia-se como satisfatório o aprendizado proporcionado, e valioso quanto a exposição das dificuldades reais de implementação do BIM. Futuras aplicações podem analisar outras sequências de exposição dos conceitos básicos previamente ao desenvolvimento de projetos, o que pode aumentar a absorção inicial de conteúdos e a velocidade de trabalho.

Como proposta de melhoria para a próxima aplicação da atividade pedagógica, sugere-se a aplicação de um questionário anteriormente ao desenvolvimento dos produtos e ao fim do processo, para melhor avaliar e registrar a evolução do aprendizado dos alunos, indo além das impressões qualitativas ao longo da atividade. Acredita-se que é preciso deixar claro aos alunos que o questionário apenas servirá para avaliação interna, e que o ambiente da disciplina deve ser de aprendizado e colaboração. Por esse mesmo motivo, não é necessário que os alunos possuam conhecimento prévio para participar da atividade, já que sua intenção principal é proporcional aprendizado prático e refletir a real complexidade de trabalhar em BIM. Outra proposta seria a redução do número de fases, para ofertar mais tempo de trabalho às fases restantes e mais absorção de conhecimento.

De forma mais abrangente, sugere-se a revisão do plano pedagógico dos cursos de Engenharia Civil para a introdução de disciplinas integradas que promovam o trabalho em equipe e o entendimento da interdependência. Ainda que não tenham como objetivo tratar sobre o processo BIM, a simples inserção de atividades colaborativas introduzem conceitos básicos e proporcionam um maior envolvimento do aluno em todo o curso. Além disso, a inserção de uma metodologia ativa à nível de graduação que incentive o desenvolvimento de habilidades de comunicação, cooperação, compatibilização e fluxo de informações reforça também a necessidade de ensinar conhecimentos além dos livros. A experiência com procedimentos intangíveis é essencial para que os profissionais recém-formados se sintam mais seguros de suas habilidades no mercado de trabalho e para melhor se adaptarem a qualquer ambiente. 


\section{Referências}

[1] M. B. Ribeiro, M. C. Gonçalves and P. M. Magalhães, "Implementação do modelo de informação integrado no BIM em obra ferroviária", in $1^{\circ}$ Congresso Português de Building Information Modelling (2016), Universidade do Minho, pp. 99-108, Guimarães - Portugal, 2016. doi: 10.5281/zenodo.166758.

[2] S. Azhar, M. Khalfan and T. Maqsood, "Building Information Modeling (BIM): Now and Beyond”, Australasian Journal of Construction Economics and Building, 12(4(, pp. 15-28, 2012. doi: 10.5130/AJCEB.v12i4.3032.

[3] A. Silva, D. Drummond and J. Oliveira, "BIM A400: Implementação, resiliência, rentabilidade", in $1^{o}$ Congresso Português de Building Information Modelling (2016), Universidade do Minho, pp. 19-32, Guimarães - Portugal, 2016. doi: 10.5281/zenodo.166758.

[4] R. Owen, R. Amor, J. Dickinson, M. Prins and A. Kiviniemi, "Research Roadmap Report: Integrated Design \& Delivery Solutions (IDDS)", in International Council for Research and Innovation in Building and Construction (2013), 37 p., CIB Publication 370, ISBN 978-90-6363-072-0.

[5] J. C. Lino, N. Lacerda, F. Reis and B. Caires, "Soluções para a coordenação, implementação e gestão BIM - uma perspectiva global", in $1^{\circ}$ Congresso Português de Building Information Modelling (2016), Universidade do Minho, pp. 33-43, Guimarães Portugal, 2016. doi: 10.5281/zenodo.166758.

[6] M. Pires and A. Monteiro, "Implementação BIM na CASAIS S.A.", in $1^{o}$ Congresso Português de Building Information Modelling (2016), Universidade do Minho, pp. 69-76, Guimarães - Portugal, 2016. doi: 10.5281/zenodo.166758.

[7] P. Serra, C. Canelhas, A. Raposo and J. Cidades, "Implementação do conceito BIM na COBA", in $1^{\circ}$ Congresso Português de Building Information Modelling (2016), Universidade do Minho, pp. 57-67, Guimarães - Portugal, 2016. doi: 10.5281/zenodo.166758.

[8] A. Monteiro, J. Lima, S. Henriques, L. Rodrigues and L. Ribeirinho, "Implementação BIM numa empresa de estudos e projetos de Engenharia (CENOR)", in $1^{\circ}$ Congresso Português de Building Information Modelling (2016), Universidade do Minho, pp. 77-85, Guimarães - Portugal, 2016. doi: 10.5281/zenodo.166758.

[9] J. C. Lino, M. Azenha and P. Lourenço, "Integração da Metodologia BIM na Engenharia de Estruturas", in Encontro Nacional BETÃO ESTRUTURAL - BE2012, Faculdade de Engenharia da Universidade do Porto (FEUP).

[10] R. Sacks, and R. Barak, "Teaching Building Information Modeling as an Integral Part of Freshman Year Civil Engineering Education", Journal of Professional Issues in Engineering Education, 136(1), pp. 30-38, 2010. doi: 10.1061/(ASCE)EI.19435541.0000003.

[11] M. B. Barison and E. T. Santos, "Ensino BIM: tendências atuais no cenário internacional", Gestão e Tecnologia de Projetos, vol. 6, n. 2, pp. 67-80, 2011. doi: 10.4237/gtp.v6i2.218.

[12] R. C. Ruschel, M. L. V. X. Andrade and M. Morais, "O ensino de BIM no Brasil: onde estamos?”, Ambiente Construído, 13(2), pp. 151-165, 2013.

[13] R. C. Ruschel, "To BIM or not to BIM?", in III Encontro da Associação Nacional de Pesquisa e Pós-graduação em Arquitetura e Urbanismo - ENANPARQ, São Paulo, 2014. 
[14] A. L. Y. Ito and S. Scheer, "Um levantamento em Curitiba das percepções do potencial do BIM no ensino em cursos de Arquitetura", in $1^{\circ}$ Simpósio Brasileiro de Tecnologia da Informação e Comunicação na Construção - SBTIC, pp. 308-315, Fortaleza - Ceará Brasil, 2017.

[15] M. L. V. X. Andrade, L. T. M. Mendes and I. Eloy, "BIM na concepção do projeto: uma experiência didática em disciplina de Pós-Graduação", in II Encontro Nacional sobre o ensino de BIM, Fortaleza - Ceará - Brasil, 2019.

[16] R. B. Oliveira, R. O. V. R. Silva, G. M. L. Martins, R. P. Castro and M. T. M. Carvalho, "Experiência na disciplina inaugural da pós-graduação 'BIM Expert - Projetos de Infraestrutura' da UnB", in II Encontro Nacional sobre o ensino de BIM, Fortaleza, 2019.

[17] J. S. Böes and F. D. P. Lima, "BIM como metodologia ativa interdisciplinar: uma experiência de Projeto Integrador", in II Encontro Nacional sobre o ensino de BIM, Fortaleza - Ceará - Brasil, 2019.

[18] J. P. Giesta and T. G. Costa, "Experiência de inserção de conteúdos BIM na disciplina de Construção Civil”, in II Encontro Nacional sobre o ensino de BIM, Fortaleza - Ceará Brasil, 2019.

[19] D. Vieira, B. Amorim, I. Carneiro and F. Gonzaga, "As parcerias universidade-empresas como inserção do BIM na grade curricular de Engenharia Civil", in II Encontro Nacional sobre o ensino de BIM, Fortaleza - Ceará - Brasil, 2019.

[20]B. Succar, "Building Information Modelling Framework: a research and delivery foundation for industry stakeholders", Automation in Construction, 18, pp. 357-375, 2009. doi:10.1016/j.autcon.2008.10.003.

[21]A. Hermund, "Building Information Modeling in the Architectural Design Phases And Why Compulsory BIM Can Provoke Distress Among Architects", in 27th Conference on Education and Research in Computer Aided Architectural Design in Europe, pp. 75-81, Istanbul, Turkey, 2009.

[22] S. Siebelink, J. T. Voordijk and A. Adriaanse, "Developing and Testing a Tool to Evaluate BIM Maturity: Sectoral Analysis in the Dutch Construction Industry", Journal of Construction Management in Engineering, 144(8), 2018. doi: 10.1061/(ASCE)CO.19437862.0001527.

[23]Z. Turk, "Ten questions concerning building information modelling", Building and Environment, 107, pp. 274-284, 2016. doi: 10.1016/j.buildenv.2016.08.001

[24] Câmara Brasileira da Indústria da Construção, "Fundamentos BIM - Parte 1: Implementação do BIM para Construtoras e Incorporadoras”, (2016), 124p., CBIC Publication.

[25] Câmara Brasileira da Indústria da Construção, "Fundamentos BIM - Parte 2: Implementação do BIM para Construtoras e Incorporadoras", (2016), 124p., CBIC Publication. 